\title{
LA PROBLEMÁTICA DE LA CONSERVACIÓN DE OBRAS CONTEMPORÁNEAS DE CARÁCTER INMATERIAL, INSTALATIVO Y TECNOLÓGICO
}

\section{Imanol Sánchez Díez}

Universidad del País Vasco/Euskal Herriko Unibertsitatea. Dpto. Escultura y de Arte y Tecnología

\section{Resumen}

Desde finales del siglo XX la producción artística no implica únicamente la producción de objetos. El hacer artístico contemporáneo también acoge obras de carácter vivencial, inmaterial y vinculado con la acción, cuyo interés reside no tanto en el producto matérico generado por el artista, sino en la experiencia de quien participa en la obra. Es por ello que surge una paradoja a la hora de establecer unas pautas para poder mantener y conservar ciertas obras que no constan de un entendimiento clásico sobre el proceso de conservación asociado a la materia y la perduración de la obra contra el tiempo. El presente texto analiza las características de la producción artística inmaterial e identifica las responsabilidades implícitas que deben de desarrollar los y las creadoras. Con la intención de ofrecer un nuevo replanteamiento sobre esta problemática, se ofrecen alternativas y soluciones para la conservación de las obras inmateriales desde la perspectiva tanto de los artistas como de los conservadores/as y restauradores/as de arte actuales.

\section{Palabras clave: ARTE INMATERIAL; ARTE CONTEMPORÁNEO; ARTE TECNOLÓ- GICO; CONSERVACIÓN; RESTAURACIÓN}

\section{THE PROBLEM OF THE CONSERVATION OF CONTEMPORARY WORKS OF AN IMMATERIAL, INSTALLATION-BASED AND TECHNOLOGICAL NATURE}

\begin{abstract}
Since the end of the 20th century, artistic production has not only involved the production of objects. Contemporary art also includes works of an experiential, immaterial and performative, whose interest lies not so much in the material product generated by the artist, but in the experience of those who participate in the artwork. This is why a paradox arises when it comes to establishing guidelines for the maintenance and conservation of certain artworks that do not have a classical understanding of the conservation process associated with the material and the durability of the artistic work over time. This text analyzes the characteristics of immaterial artistic production and identifies the implicit responsibilities that creators must develop. With the intention of offering a new approach to this problem, it offers alternatives and solutions for the conservation of immaterial artworks from the perspective of both artists and today's art conservators and restorers.
\end{abstract}

Keywords: INTANGIBLE ART; CONTEMPORARY ART; TECHNOLOGICAL ART; CONSERVATION; RESTORATION

\footnotetext{
Sánchez Díez, Imanol. 2021. "La problemática de la conservación de obras contemporáneas de carácter inmaterial, instalativo y tecnológico". AusArt 9 (2): 31-46. DOI: 10.1387/ausart.23088
} 


\section{CAMBIO EN LA CONCEPCIÓN DEL TÉRMINO CONSERVACIÓN DESDE LA DISOLUCIÓN DEL OBJETO ARTÍSTICO}

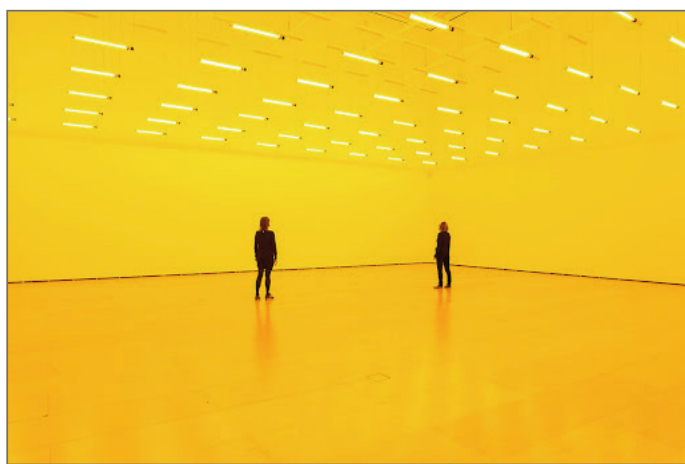

Room for one colour de Olafur Eliasson, 1997.

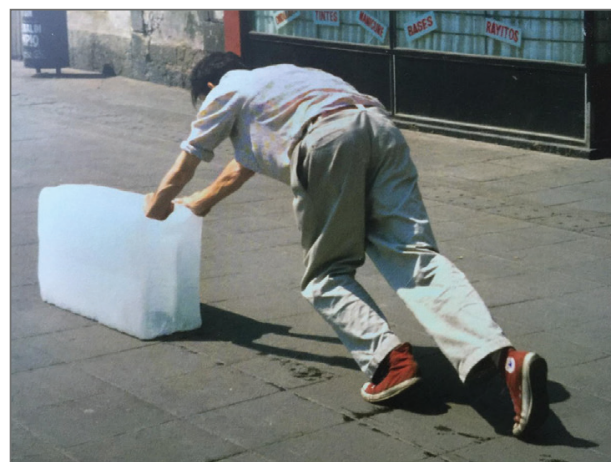

In Sometimes Making Something Leads to Nothing de Francis Alÿs, 1997.

La conservación de producciones artísticas contemporáneas genera problemáticas y planteamientos nuevos, comúnmente asociados a la configuración formal. Desde los años sesenta del siglo anterior, podemos observar cómo el objeto artístico se disuelve exponencialmente. Tal y como dice Mario Perniola (2012), "la perspectiva del restaurador y del conservador se centra en la obra que debe preservarse de la degradación provocada por el paso del tiempo (...) El artista, en cambio, comienza a ver en el arte algo más que un artefacto". Destacan, de este modo, los proyectos cuyo enfoque es puramente conceptual, como se puede observar en las distintas obras comprendidas dentro del denominado action art, tales como In Sometimes Making Something Leads to Nothing (1997) de Francis Alÿs o la obra Conversaciones telefónicas (1973) de Isidoro Valcárcel Medina; y también en aquellas creaciones donde se genera una atmósfera para establecer ciertos estímulos que los visitantes puedan experimentar, como en los casos de Room for one colour, (1997) de Olafur Eliasson o D-N SF 12 PG VI 14 (2012) de Douglas Wheeler. Es decir, muchos artistas se desvinculan del arraigo a lo matérico y, con ello, surge una problemática a la hora de establecer la conservación de aquellas obras que tienen ese carácter inmaterial. Por ello, si aplicamos el significado de los términos conservación y restauración a este tipo de obras, se abre un debate sobre a qué aluden y qué acciones concretas están vinculadas a su ejercicio. De esta manera, se pretende establecer una dirección donde estos conceptos estén más cerca de la reformulación, recreación o la reconstrucción de las obras, sobre todo, si su núcleo conceptual se centra en ser visualizadas, exploradas 
e interpretadas presencialmente. La recreación "permite reproducir o rehacer, una obra perceptualmente idéntica" y es una labor de conservación y restauración "que permite contemplarla como si hubiese sido concebida originalmente" (García Morales \& Montero 2010).

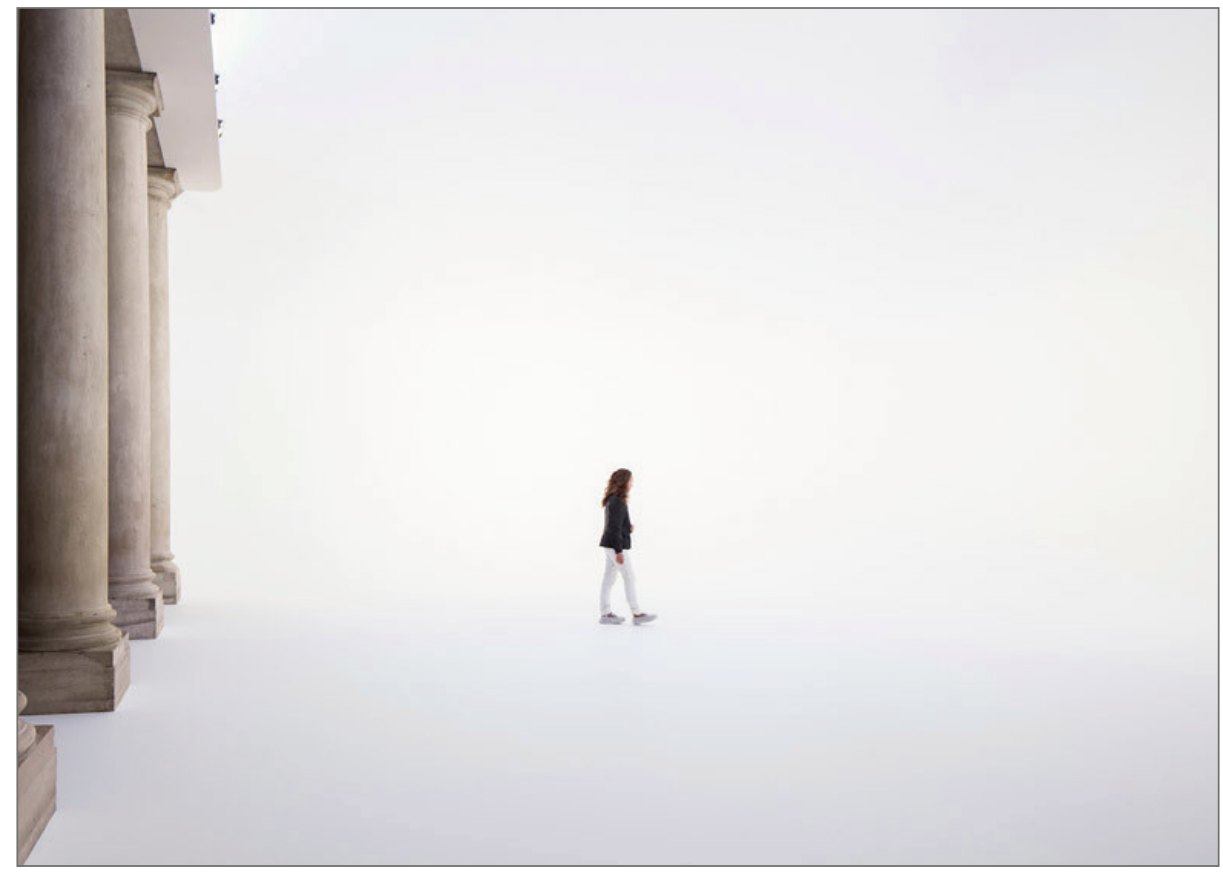

D-N SF 12 PG VI 14, 2012 de Douglas Wheeler.

\section{EL REGISTRO Y LOS VESTIGIOS DE LOS EVENTOS ARTÍSTICOS}

Muchas de las creaciones existen únicamente en formato de acción mientras son exhibidas. Después se desvanecen. Comúnmente quedan los vestigios de su hacer y registros en fotografía, video, esquemas, dibujos o transcripciones. Un ejemplo claro de ello son los apuntes para las instalaciones Landscape for Fire (1972) o Face to face (2013) de Anthony McCall. Una vez que el evento

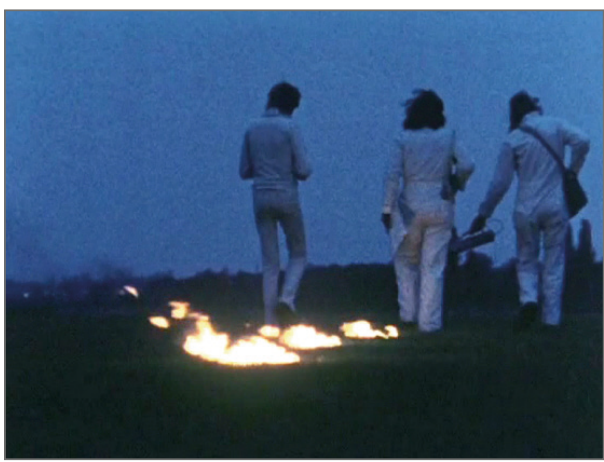

Landscape for Fire de Anthony McCall, 1972. 
termina, el público puede visualizar y entender la acción realizada, pero su análisis y reflexión es a través de todo este tipo de registros que hablan de la obra. El potencial y el interés de las producciones con estas características reside, precisamente y en gran medida, en ese carácter perecedero en el tiempo. Del mismo modo sucede con las obras de acción, también llamadas happening. "La acción del arte no consiste ya en la fabricación de un artefacto que pueda identificarse como obra artística, sino en una acción, a veces ínfima (...) Ser artista se convierte en un especie de actitud para la acción" (Perniola 2012).

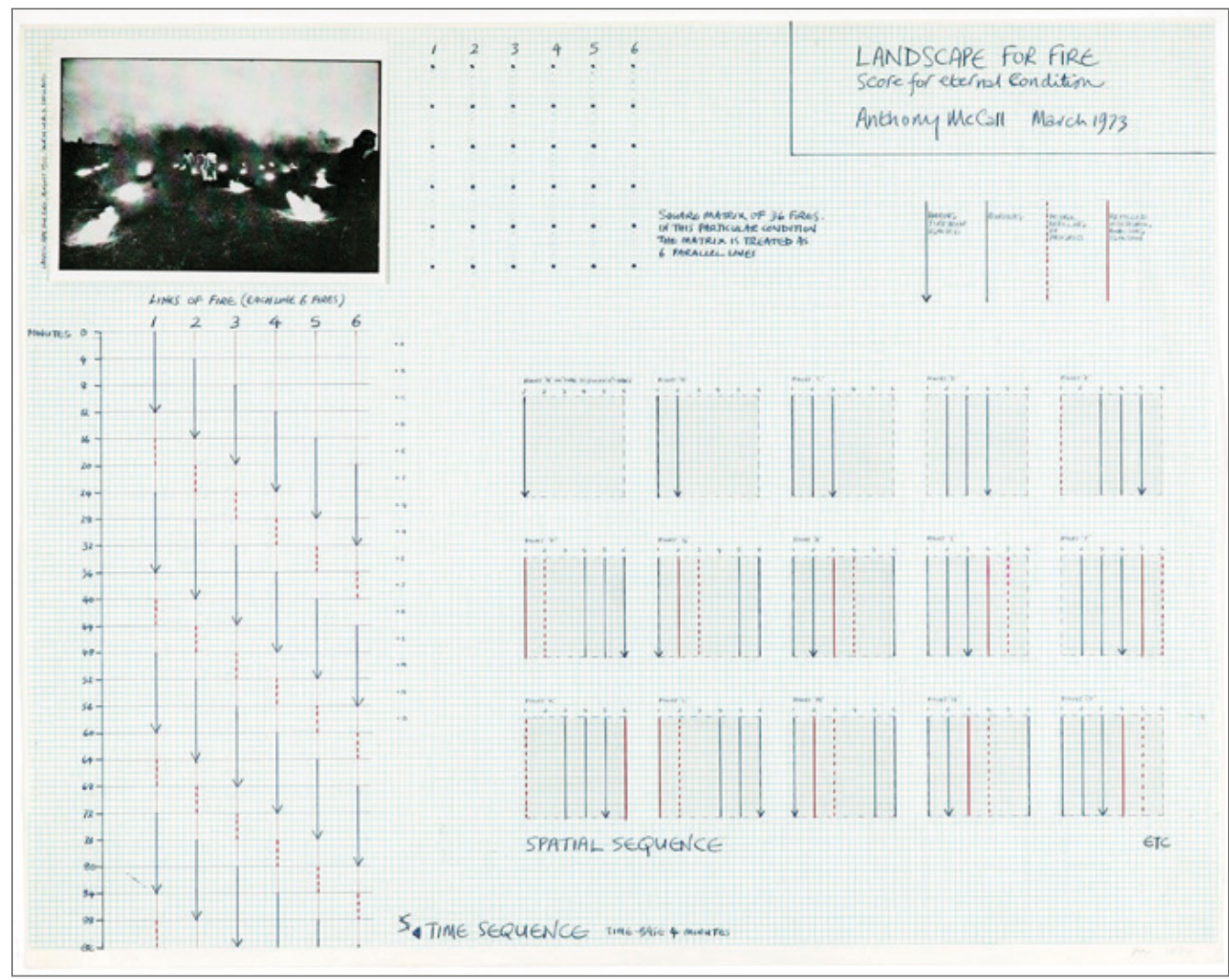

Anotaciones para Landscape for Fire de Anthony McCall, 1972.

Este material de archivo enriquece la obra, aporta diferentes puntos de vista y ahonda en la creación. McCall por ejemplo guarda cuadernos de trabajo ordenados secuencialmente y fechados meticulosamente. Desde principios de la década de 1970, McCall ha acumulado, registrado y archivado alrededor de 150 cuadernos. Estos cuadernos contienen "diagramas, guiones gráficos, 
bocetos, planos, proyecciones, registros, inventarios, mapas, borradores, anotaciones, garabatos", así como "instrucciones, notas para sí mismo, reflexiones, revisiones de revisiones y referencias" (Ellard \& Johnstone 2015). De modo que también sirven para mostrar el proceso, ideas previas, ensayos y otro tipo de documentación. Todas estas ramificaciones de la obra aportan información útil, expanden el conocimiento acerca de la tipología de la obra y pueden ser de gran ayuda para su examinación. Definitivamente, sirven para su con-

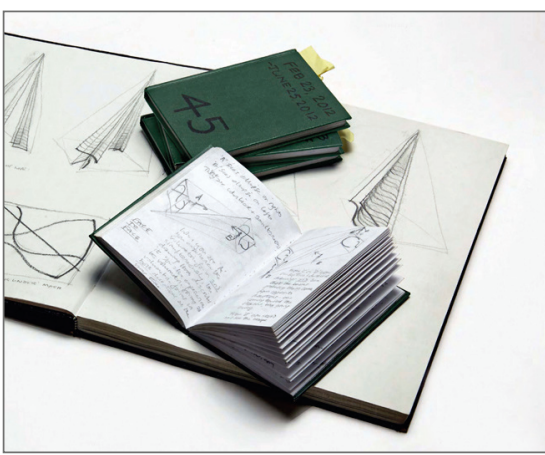

Cuadernos de Anthony McCall. servación y difusión, pero nunca suplantan a la obra como tal. Se conciben como parte integrante del universo que gira en torno a la producción artística.

Si no es necesaria la participación de la figura como artista o colectivo durante el evento (en muchas obras performáticas o instalaciones con visuales en vivo, por ejemplo, es fundamental su participación), cabe la posibilidad de que los y las creadoras establezcan unas directrices a seguir. Es decir, si se manifiesta expresamente y se pauta la configuración metodológica, las necesidades, los parámetros espaciales requeridos, los pasos a seguir, etc... estos pueden ser reproducidos por otra persona o instituciones, de forma que esta nueva reproducción de la obra vaya acorde a los intereses originales del artista. Si la carga conceptual reside en la visualización de la obra en vivo, es comprensible que los y las artistas tengan el propósito de exhibir la obra tal y como fue concebida, evitando así su muestra a modo de registro. Por lo tanto, se abre la posibilidad de rehacer la obra, lo que implica un tipo de conservación basada en el mantenimiento y exhibición al público.

Esta opción implica la consciencia por parte de los y las creadoras en establecer y determinar cómo quieren y deciden conservar su su obra. Existen procedimientos y herramientas que facilitan a los artistas esta tarea. En junio de 2003, la Fundación Daniel Langlois y el Museo Guggenheim lanzaron el Variable Media Questionnaire (http://variablemediaquestionnaire.net/), una herramienta informática iniciada por Jon Ippolito, y más tarde desarrollada por Caitlin Jones y Alain Depocas. Es un cuestionario interactivo que funciona como base de datos y sirve para ayudar a artistas y conservadores a solventar 
los posibles problemas a la hora de exhibir y conservar sus obras. La iniciativa tiene como objetivo definir cada uno de estos estudios de caso en términos de comportamientos independientes del medio e identificar estrategias aprobadas por los artistas para preservar las obras de arte con la ayuda del cuestionario interactivo". El propio Ippolito señala que este cuestionario "no es tanto una investigación sociológica como un instrumento para determinar cómo a los artistas les gustaría que su trabajo fuera recreado en el futuro llegado el caso" (citado en García-Morales \& Montero 2010).

Existen otras vías para mantener la voluntad de los y las artistas presente, archivada y almacenada como es el caso de la Tesis; La entrevista al artista emergente como modo de conservación preventiva de la doctora Ruth del Fresno Guillem. En ella establece "unas pautas de conservación preventiva tempranas y seguras, que respeten la intencionalidad y el concepto de deterioro de cada uno de los artistas, a través de la información extraída por el uso de la entrevista" (2017). La conversación directa con los creadores, establece de primera mano los pensamientos y pretensiones acerca de cómo determinan cada uno su propia producción.

La realización de las entrevistas, la creación de documentos escritos y participación en cuestionarios son acciones que se establecen como un trabajo integrado en el desarrollo conceptual de los y las artistas, que facilitan estas alternativas para la correcta conservación de su obra. Al mismo tiempo, deja latente su interés por mantener la exposición abierta a los visitantes, permitiendo de esta forma la posibilidad de su exploración o la experiencia directa. Es así como actualmente un gran peso de la conservación recae directamente sobre el posicionamiento conceptual de los y las creadoras.

\section{LA CONSERVACIÓN EN LA ACTUALIDAD Y LA FIGURA DEL CONSERVADOR/A COMO MEDIADOR/A}

Hoy en día, la conservación no implica únicamente incidir sobre el aspecto físico y químico de la obra; es decir, sobre los materiales, la limpieza, u otros aspectos como la temperatura. Actualmente, la conservación también es necesaria en la reproducción de ciertas obras que conllevan el montaje íntegro de la instalación, o eventos artísticos dirigidos a través de las pautas establecidas 
por su autor o autora. En este caso, la conservación se basa en recrear y reacondicionar una réplica, respetando su carácter inicial sin la necesidad de la presencia del artista. Tal y como dicen García Morales y Montero Vilar (2010), en este sentido, la terminología 'original', 'copia' y 'réplica' adquieren nuevos significados. Considerando que 'original' es la obra de la que no se han hecho copias, y que 'copia' se designa a aquella repetición que busca la máxima semejanza posible de la obra por alguien ajeno al creador, en el arte digital, y al mismo tiempo en el inmaterial e instalativo, "se hablaría de ediciones o de réplicas, a aquellas otras realizadas por el mismo autor".

En cuanto al rol de la figura del agente que conserva y restaura obras artísticas, este puede establecerse como un mediador entre los artistas y la institución. Tal como comentan Flores de Medeiros y Llamas Pacheco, una buena manera de afrontar la conservación es establecer una comunicación con el autor o autora, teniendo en cuenta tanto su idea original como también los condicionantes de la institución para encontrar así posibilidades que lo hagan factible. Para ello, inciden en que es fundamental "que la institución tenga claras sus condiciones estructurales, técnicas y de personal antes de adquirir una obra". Así mismo subrayan "la posibilidad de plantear una charla con el artista para buscar un punto de equilibrio entre sus deseos y la viabilidad de mantenimiento y re-exhibición de una obra". (Flores de Medeiros \& Llamas 2017). Los documentos generados por los y las artistas y mediadoras para el mantenimiento o muestra al público sirven para que su obra perdure en el tiempo, incluso cuando los o las creadoras hayan fallecido. De este modo, la conservación del arte contemporáneo incluye "la coordinación y asesoramiento en los desafíos que muchas veces plantean las obras de arte o las necesidades del artista", trabajando directamente con ellos, además de la labor de recolección de información. (García González 2009).

Se deben de tener en cuenta, por otra parte, las instalaciones site specific con una durabilidad limitada. Si las instalaciones perecederas generan la problemática en cuanto la durabilidad en el tiempo, puede sumarse una condición relacionada con la espacialidad, que dificulta aún más el proceso de reconstrucción. Una vez finalizado el evento, si se diera el caso de volverse a mostrar tal y como lo pudo visitar el público con anterioridad y si el o la artista no muestra la posibilidad de ser flexible con las dimensiones de su obra, su montaje queda anclado a un emplazamiento en concreto o a unas medidas específicas. Una solución posible es adecuar el espacio expositivo con falsas paredes para ceñirse al display original. Es decir, integrar las dimensiones y formas de la arquitectura original, dentro de un espacio mayor. De esta forma, 
la obra podría ser expuesta en diferentes centros, instituciones o museos, pero en lo que se refiere al espacio concreto que ocupa la instalación sería exactamente igual.

Por otra parte, la alteración de las formas de la sala expositiva también es parte del proceso de creación de algunos artistas. Ejemplo de ello es la serie titulada Space Division Constructions (1976-2013) de James Turrell. Son instalaciones en las que inicialmente no se aprecia una división espacial, gracias al alto contraste de intensidad y color de las luminarias. Normalmente una de las paredes tiene un rectangulo de color, el cual en realidad es un orificio que da acceso a un nuevo habitáculo. Cuando el visitante entra en la sala, inicialmente considera que ese rectángulo de color es una superficie plana. Esta manipulación perceptiva "se consigue con la iluminación homogénea por varias luces del mismo color y las curvaturas de las aristas para favorecer la pérdida de los límites espaciales" (Sánchez 2020). Turrell muestra la pretensión de "no utilizar ningún objeto porque no quiere tener la luz alumbrando cosas, quiere cosificar la luz". Por ello, no existe objeto, ya que "la percepción de la luz es el objetivo y la percepción es el objeto" (Turrell 1992).

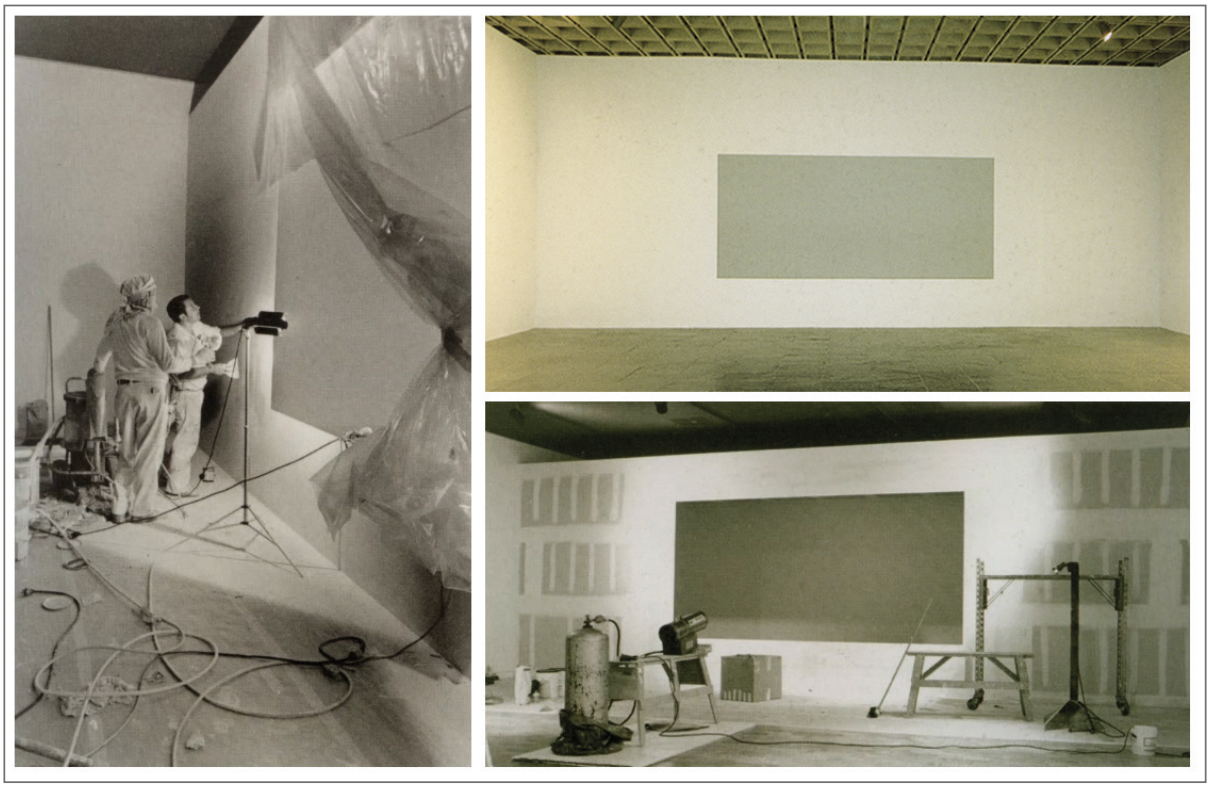

Construcción de Space Division Constructions de James Turrell, 1976-2013.

También hay que considerar cuestiones relacionadas con derechos, exclusividad y las licencias permitidas acordadas entre el o la artista, la institución o los agentes que adquieren o son beneficiarios de esas licencias. La producción 
de la instalación, evento expositivo o acción artística dependerá de la combinación de estos acuerdos.

\section{LA INCORPORACIÓN DE LOS DISPOSITIVOS TECNOLÓGICOS EN LA CONSERVACIÓN}

La tecnología y su integración en el arte también supone un nuevo dilema. Las obras creadas con materiales tecnológicos dependen de su obsolescencia, pero por lo general estos elementos funcionan como herramienta para producir algo. Es decir, la mirada de los visitantes no se centra en los artilugios que forman parte de la producción artística (proyectores, luminarias, sensores, chips, CPUs...), sino en lo que se crea a través de la utilización de estos dispositivos. Así, su reemplazamiento por uno del mismo estilo puede modernizar la instalación sin que se altere el aspecto o el contenido conceptual. En caso de rotura o pérdida del dispositivo, el reemplazamiento garantiza una conservación óptima, evitando así que la obra sufra cualquier alteración. García-Morales y Montero Vilar mencionan que "la obsolescencia de los soportes digitales es lo que puede fallar, lo que envejece" y que asimismo, "es un medio y no un fin por lo tanto, susceptible de asimilar una intervención restauradora, incluso una sustitución". (García Morales \& Montero 2010). Para ellas, esta posibilidad está próxima al concepto de restauración arquitectónica, donde la restauración puede realizarse con materiales diferentes al original, pero por ello no son menos auténticos en el papel que desempeñan. Si hubiera información digital, una buena opción es tener las copias de seguridad también en espacios virtuales. Por ello cabría valorar "la creación de bases de almacenamiento de datos y centros de documentación accesibles", ya que "toda la información generada debe ser analizada, catalogada y conservada y además esta debe ser accesible a investigadores e historiadores del arte". (García González 2009).

La posibilidad de suplantar las herramientas permite el desplazamiento geográfico de la instalación artística de forma más rápida y con mayor seguridad. Dado que la obra no está condicionada por ser un elemento físico singular, como un objeto, cuadro o escultura única, esto abre vías para su traslación a espacios diferentes sin riesgos. Si el o la artista lo quisiera, puede exponer la misma obra simultáneamente en diferentes lugares, lo que implica la posibilidad de que su obra tenga un mayor alcance y, por lo tanto, esta pueda ser experimentada en diferentes partes del mundo por múltiples personas. En 
cuanto a la entidad financiadora que cuente con las licencias de exposición, estas puede sacar partido económico, ya que el gasto es menor si el artista acepta que las herramientas empleadas puedan ser alquiladas, evitando de esa manera tanto la compra del material como el embalaje, desplazamiento y los seguros que supone cuando se está tratando con una pieza única. Además, tal y como comenta Alicia García González (2009) los centros y "museos de arte contemporáneo tienen la responsabilidad de estudiar, divulgar y conservar su colección", ya que hoy en día, "se han convertido en un foco generador de arte y una aportación fundamental para su estudio".

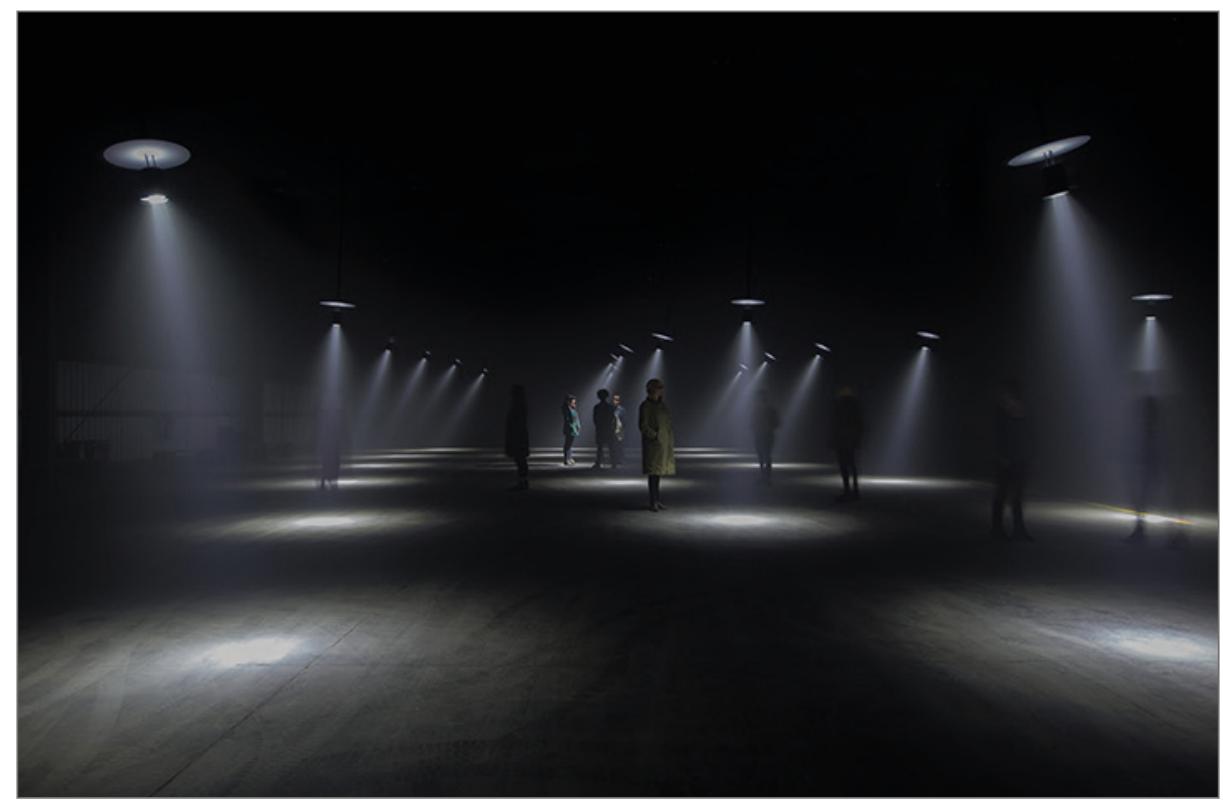

Our Time de United Visual Artists, 2016.

En el caso de que los elementos integrados que componen la instalación sean únicos, es decir, que sean material tecnológico creado expresamente por el o la artista para una acción concreta, se requiere una conservación similar al proceso clásico de la restauración artística. Es el caso de la instalación Our Time (2016) del colectivo UVA en donde 21 luminarias construidas por los artistas están divididas en tres filas. Estos dispositivos tienen simultáneamente dos tipos de iluminación de diferentes intensidades y a raíz de la propia construcción la textura es diferente. Además están vinculadas con una CPU para poder realizar un movimiento ondulatorio. Si se tuvieran que arreglar o reemplazar estas luminarias, al no provenir de fábrica, existen, principalmente, dos opciones. Puede que se restaure y conserve a través de la determinación 
de técnicos por evaluación propia, o puede que sea posible reparar o reproducir nuevamente el dispositivo siguiendo esquemas y medidas establecidas por el o la artista. Si bien es cierto que, mediante su observación, los expertos podrían encontrar su propia fórmula para la reproducción del dispositivo, el hecho de que los o las creadoras hayan decidido dejar un documento técnico sobre su construcción implica que se ocupan de su conservación y contemplan la importancia de la construcción de dicha obra para mantener su sentido original.

\section{LA CONSERVACIÓN Y REPRODUCCIÓN DE OBRA ARTÍSTICA FUNDAMENTADA EN INSTALACIONES ATMOSFÉRICAS}

Desde la experiencia como productor artístico e investigador de obras fundamentadas en la luz, se han generado situaciones donde ha sido obligatoria la toma de decisiones respecto a su formato de cesión, venta y conservación. En el caso concreto de las instalaciones atmosféricas y lumínicas, su particularidad reside en trabajar el espacio con un elemento inmaterial y polivalente como es la luz. Esta se utiliza por su capacidad para generar diferentes percepciones y conseguir, a través de ella, diversos estímulos que el público pueda experimentar. Lo más destacable de la luz, es que es el mayor condicionante del lugar intervenido. Esta cualidad es el motor que permite que esas percepciones se generen durante la vivencia del visitante.

Se determinan instalaciones atmosféricas, aquellas obras artísticas que "condicionan un espacio para configurar un ambiente concreto y que, además, otorgan al individuo la capacidad de significar la obra en base a su experiencia”. (Sánchez, 2020). De esta forma la presencia y vivencia del público es esencial, a tal punto que se define como inacabada una obra que no es vivida por los visitantes. Como menciona Carles Méndez Llopis,"en los años 70, las dimensiones de las piezas eran transitables", y el sujeto era capaz de circular libremente por el espacio, lo que le permitía vivir la obra. Más allá de lo visual, "el environment obligaba a cierta participación, a practicar el espacio y sus objetos por medio de la exploración" (Llopis 2013). Esta condición siempre remite a la vinculación público-espacio-instalación; es decir, a la asistencia dentro de los parámetros físicos y espaciales en donde se encuentra la atmósfera o los estímulos creados. Es así cómo estas instalaciones necesitan 
"mayor responsabilidad, atención y participación del sujeto, que es el que finalmente construye la obra" (Méndez Llopis 2013). Por ello, una vez concluido el evento expositivo, la obra desaparece.

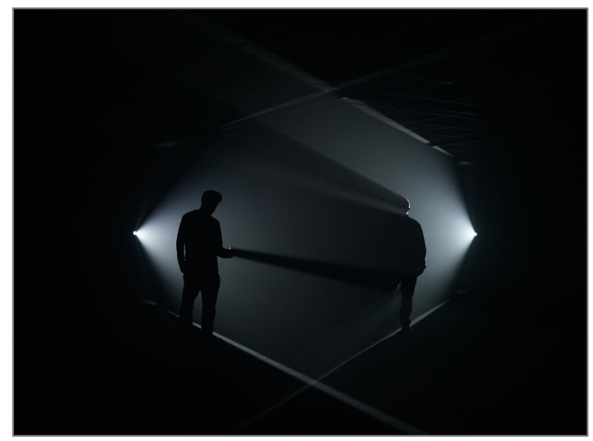

Light, water; Ganzfeld de Imanol Zubiauz, 2020.
Cuando se vuelve a generar esa misma obra, surge una reformulación que permite adaptar la misma instalación a otra estancia con dimensiones y formas arquitectónicas diferentes. Asimismo, las condiciones ambientales también son susceptibles a su modificación; por lo tanto, es difícil delimitar en dónde se produce la conservación de una instalación atmosférica de carácter lumínico.

\section{EL REGISTRO Y ARCHIVO DE OBRAS ARTÍSTICAS FUNDAMENTADAS EN INSTALACIONES ATMOSFÉRICAS}

En el caso del registro a través de fotografías y vídeo se genera una paradoja. La obra pierde su objetivo cuando es mostrada únicamente a través de la documentación. Pese a entenderlo como una forma válida para ser mostrada, por ejemplo, a través de Internet, en catálogos o ponencias, no deja de ser una prueba de algo que existió mientras fue experimentada por el público. Al tratarse de obras cuya intencionalidad reside en la lectura de los estímulos y percepciones surgidas a través de la vivencia in situ de los visitantes, esta experiencia cambia completamente si la obra es visionada únicamente desde el registro fotográfico y videográfico. Por ello, de ninguna manera puede compararse el registro a la experimentación de las personas que puedan visitar la obra de forma física.

Es por ello que, cuando no queda otra opción debido a la imposibilidad espacial de realizar la obra de una forma óptima, además de las fotos y vídeo del registro se muestra otro tipo de material: maquetas, bocetos o luminarias despiezadas para mostrar al público la producción y el proceso. De esta manera, cuanta más información reciba el público, mayor grado de comprensión podrá tener, y con ello, generar en su mente una idea de lo que sería estar dentro de 
esa instalación atmosférica. Con esa suma de conocimientos, los visitantes pueden imaginar el tipo de percepciones y sensaciones que puede sentir una persona que sí la ha vivido presencialmente.

A la hora del almacenaje, cesión o compra, su carácter inmaterial también supone un problema. Para ejemplificarlo, se alude a la experiencia personal cuando llegó la necesidad de tomar una decisión concreta en el momento de ceder una obra atmosférica y lumínica a una institución. En este caso, derivó en crear una caja o archivador donde guardar diferente documentación. Es decir, finalmente se recurre a un elemento físico, donde poder incluir todo tipo de información.

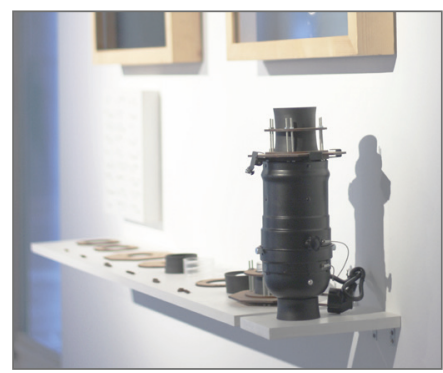

Despiece de Embody de Imanol Zubiauz, 2018.

En su tapa, grabado a láser, se muestran los datos como el título, año y autoría. Así, se incluyen las instrucciones de montaje impresas en papel, esquemas, renders, fotografías de la obra establecida con anterioridad, otras fotografías sobre el orden de montaje de las luminarias y su diseño vectorial. También se hace alusión a las condiciones ambientales y lumínicas del espacio expositivo, así como otras notas sobre la adecuación de las paredes del espacio y las dimensiones óptimas de la sala expositiva. Junto a este ejemplar impreso, se adjunta un pen drive, el cual muestra dos carpetas que incluyen los datos impresos en un archivo PDF, y otra carpeta con el archivo referente al software (libre) que se debe emplear, listo para ejecutar y que funcione automáticamente. La decisión de usar, en este caso, un software de interacción libre es debido a la facilidad de su empleo.

Junto con toda la documentación mencionada, se adjuntó el embalaje de los dispositivos específicos creados para el funcionamiento de la instalación que son únicos (sensores, luminarias y difusores con modificaciones). En cuanto a material reemplazable (proyectores, ventiladores, máquinas de humo...), se añadió un listado de los requisitos y características que deben tener. De este modo, salvo las luminarias creadas específicamente, el resto del material se puede reemplazar por uno nuevo y los elementos perecederos como el líquido que conforma la neblina que impregna el espacio, debe de ser recompuesto a medida que se gaste. 
Todo el proceso de preparación de la documentación conlleva una dedicación exclusiva para la conservación de la manera en la que ha sido producida. Por otra parte, ser consciente del grado de dificultad técnica y los posibles problemas que pueden surgir tanto a la hora de mostrar la obra como en su conservación es algo indispensable. Todo ello recala en el trabajo por dejar las instrucciones y el funcionamiento de la obra lo más simple, claro y fácil posible para los operadores.

Cabe destacar que, para el mantenimiento de las obras de luz atmosféricas, se requiere de unas instrucciones para el personal de seguridad y encargados de la sala. Aunque es preferible que la instalación sea independiente, y que se enciendan y se apaguen de forma autónoma todos los dispositivos, también es necesario que las personas encargadas del mantenimiento puedan saber cómo funcionan los dispositivos empleados. De esta forma se evitan confusiones y el creador/a se asegura que la instalación funciona de la forma adecuada durante el tiempo que se ubique en dicho espacio.

\section{CONCLUSIONES}

A medida que en el arte se produce la disolución del objeto artístico, el planteamiento para su conservación evoluciona paralelamente cambiando el rol clásico del conservador/ra, convirtiéndose en un mediador entre los creadores y los centros expositivos. Su función deriva en analizar previamente la documentación de diferentes fuentes; entrevistas registradas en audio, vídeo, conversación telefónica, e-mail, contactos directos, documentos escritos, fotografías, maquetas, croquis, dibujos... y también ha de tener en cuenta las características y limitaciones de los centros en los que se ubica la obra. Es por ello que el triángulo que se completa entre la figura de los o las artistas, el centro expositivo y los o las conservadoras, se fundamenta en la importancia de ser flexibles a la hora de establecer las condiciones y las necesidades que competen a cada uno.

Cada artista que trabaje en la producción de obras inmateriales, instalativas, tecnológicas y performativas debe de considerar una parte de su tiempo para valorar cómo se deben conservar. Puede que prefiera que su obra solo sea ejecutada durante un lapso de tiempo y que no quede más que el recuerdo de aquellos que fueron cómplices y testigos de lo sucedido. Habrá quien prefiera 
registrarlo, ya sea a través de grabaciones orales, con documentación escrita, con fotos, con vídeo o de todas esas formas simultáneamente. Lo que queda evidente es que su práctica artística conlleva una problemática respecto a su resguardo y memoria.

Comúnmente el registro es una de las partes fundamentales para mantener la memoria viva de aquellas obras que poseen un carácter de obra inmaterial. Es fundamental para la difusión on-line y sirve como huella para mostrar algo realizado. Tiene una gran utilidad en la difusión, en transmitir información y en mostrar incluso el proceso de creación, esencial para su investigación y formación didáctica de futuras generaciones. Pero nunca se debe confundir con una exposición planteada para verse presencialmente. Si se tratan de obras fundamentadas en la vivencia, debe de quedar clara la distinción entre el registro y la obra como tal.

Dada la naturaleza de la obra de carácter inmaterial, la responsabilidad de su correcta conservación y registro no solo recae sobre el conservador y restaurador de arte, sino también sobre el propio artista. La toma de posicionamiento conceptual sobre la conservación por parte del artista recaerá en el procedimiento de los profesionales a la hora de restaurar, reformular, rehacer, reinstalar o restablecer su obra. Es decir, si se establece una comunicación satisfactoria entre los artistas y los conservadores, esto beneficiará a preservar el objetivo original de dicha obra. Uno de los aspectos que cualquier artista que trabaje con la creación de arte inmaterial debería de tener presente en todo momento es que se plantee si quiere que su obra perdure en el tiempo, y si es así, pensar detalladamente en cuál es la mejor forma de hacerlo para beneficiar a su obra. Es por eso que se subraya la necesidad de que cada artista tenga un posicionamiento al respecto.

\section{Referencias bibliográficas}

Chavarria Díaz, Javier. 2002. Artistas de lo inmaterial. Hondarribia: Nerea

Ellard, Graham \& Stephen Johnstone. 2015. Anthony McCall: Notebooks and conversations. Farnham UK: Lund Humphries

Flores de Medeiros, Gilca \& Rosario Llamas Pacheco. 2017. "Projéteis: Documentación y conservación instalación”. Arché 11-12: 79-84. http://hdl.handle.net/10251/101217

Fresno Guillem, Ruth del. 2017. "La entrevista al artista emergente como modo de conservación preventiva: Estudio aplicado a los proyectos Perspectives Art Inflammation and Me y Perspectives, Art Liver Diseases and Me". Tesis Univ. Politècnica de València 
García González, Alicia. 2009. "Reflexión sobre la conservación del arte contemporáneo y su aportación a la historia del arte". Ge-Conservacion 0: 133-40. https://doi.org/10.37558/gec. v0i0.30

García Morales, Lino \& Pilar Montero Vilar. 2010. "Conservación y restauración del arte digital: Un problema conceptual”. En Conservación de arte contemporáneo: 11 Jornada. Madrid: Museo Nacional Centro de Arte Reina Sofía. https://doi.org/10.13140/RG.2.1.1302.9921

Ippolito, Jon, Richard Rinehart, Marilyn Lutz \& Sharon Fitzgerald. 2009. "Forging the future: New tools for variable media art preservation". In JCDL '09: Proceedings of the 9th ACM/ IEEE-CS joint conference on digital libraries, 403-4. New York: Association for Computing Machinery. https://doi.org/10.1145/1555400.1555484

Méndez Llopis, Carles. 2013. "El espacio de la inmaterialidad: Del ocultamiento a plena vista de Olafur Eliasson". El Artista 10: 131-9

Noguera Giménez, Juan Francisco. 1996. “Restaurar ¿Es todavía posible?”. Loggia 1: 6-15. https://doi.org/10.4995/loggia.1996.5479

Perniola, Mario. 2012. “¿Restaurar o renovar el arte contemporáneo?”. Traducción de Alexandra Bouteaux. Número monográfico "Estetización y nuevas artes", Contrastes supl. XIII: 75-185. https://doi.org/10.24310/Contrastescontrastes.v0i0.1278

Sánchez Díez, Imanol. 2020. "Tipificación y práctica de obras artísticas fundamentadas en la luz de carácter ambiental". Tesis Univ. del País Vasco / Euskal Herriko Unibertsitatea. http://hdl.handle.net/10810/51250

Turrell, James. 1992. James Turrell: Sala de Exposiciones de la Fundación "la Caixa" ... Madrid, 12 de noviembre de 1992-10 de enero de 1993. Editado por Jiri Svestka en colaboración con Alison Sarah Jacques \& Julia Brown ; introducción de Luis Monreal \& Thomas M. Messer. Stuttgart: Cantz

Worms, Vincent \& Joseph del Pesco. 2018. "Anthony McCall: Landscape for fire”. Kadist.org, 2 dic. https://kadist.org/work/landscape-for-fire/

\section{Notas}

${ }^{1}$ INCAA. 2003. International Network for the Conservation of Contemporary Art. Variable Media Network. https://www.incca.org/articles/organisation-variable-media-network 